\title{
1 Differential richness inference for 16S rRNA marker gene surveys
}

2 M. Senthil Kumar ${ }^{1,2, *}$, Eric V. Slud ${ }^{3,4}$, Christine Hehnly ${ }^{5,6,9}$, Lijun Zhang ${ }^{5,9}$, James Broach ${ }^{5,6}$, Rafael P. Irizarry ${ }^{1,2}$, Steven

3 J. Schiff ${ }^{7,+}$, Joseph N. Paulson ${ }^{8,+, *}$

4

${ }^{1}$ Department of Data Science, The Dana-Farber Cancer Institute, Boston, MA

$6 \quad{ }^{2}$ Department of Biostatistics, Harvard T.H. Chan School of Public Health, Boston, MA

$7 \quad{ }^{3}$ Department of Mathematics, University of Maryland, College Park, MD

$8 \quad{ }^{4}$ Center for Statistical Research and Methodology U.S. Census Bureau, Suitland, MD

$9 \quad{ }^{5}$ Penn State Institute for Personalized Medicine, The Pennsylvania State University College of Medicine, Hershey, PA

${ }^{6}$ Department of Biochemistry and Molecular Biology, The Pennsylvania State University College of Medicine, Hershey, PA

$12 \quad{ }^{7}$ Department of Engineering Science and Mechanics, Pennsylvania State University, State College, PA

$13{ }^{8}$ Department of Biostatistics, Product Development, Genentech, San Francisco, CA.

$14{ }^{9}$ Contributed equally.

$15{ }^{+}$Co-senior.

16 *Corresponding: MSK: senthil@ds.dfci.harvard.edu; JNP: paulson.joseph@gene.com

Preprint server: Biorxiv, link: https://www.biorxiv.org/content/10.1101/2021.11.07.467583v1

Classification. : Physical sciences > Biophysics and Computational Biology, Biological sciences > Microbiology

Keywords. : richness, species misclassification, 16S microbiome, mathematical model, amplification, sequencing 


\section{Abstract}

25 Individual and environmental health outcomes are frequently linked to changes in the diversity of 26 associated microbial communities. This makes deriving health indicators based on microbiome 27 diversity measures essential.

While microbiome data generated using high throughput 16S rRNA marker gene surveys are appealing for this purpose, $16 \mathrm{~S}$ surveys also generate a plethora of spurious microbial taxa. When this artificial inflation in the observed number of taxa (i.e., richness, a diversity measure) is ignored, we find that changes in the abundance of detected taxa confound current methods for

33 inferring differences in richness.

Here we argue that the evidence of our own experiments, theory guided exploratory data analyses and existing literature, support the conclusion that most sub-genus discoveries are spurious artifacts of clustering $16 \mathrm{~S}$ sequencing reads. We proceed based on this finding to model a $16 \mathrm{~S}$ survey's systematic patterns of sub-genus taxa generation as a function of genus abundance to derive a robust control for false taxa accumulation.

Such controls unlock classical regression approaches for highly flexible differential richness inference at various levels of the surveyed microbial assemblage: from sample groups to specific taxa collections. The proposed methodology for differential richness inference is available through an R package, Prokounter. 


\section{Introduction}

Clinically relevant health outcomes are often accompanied by changes in the diversity of associated microbial communities. For instance, decreased gut microbiome diversity accompanies childhood diarrhea ${ }^{1}$, enteric infections ${ }^{2}$, and has been shown to predict the onset of infant type I diabetes ${ }^{3}$. Distinct intra-tumoral microbial diversity levels are associated with cancer sub-types ${ }^{4-6}$. Thus, inferring disease associated changes in microbiome diversity metrics is useful for characterizing disease pathology and progression.

Among the various diversity measures, richness quantifies the number of taxonomic groups in a community ${ }^{7,8}$. Changes in species richness of biological communities have informed key environmental management practices that are relevant to public health and well-being ${ }^{7-23}$. Of the technologies available for characterizing microbial communities, 16S rRNA gene surveys are widely adopted for their high throughput and low cost. As a broad screening tool, they largely avoid the need for laborious culturing of microbes. This makes them especially attractive for deriving health metrics based on the microbiome.

In this work, we focus on inferring changes in richness of microbial communities between sample groups (i.e., differential richness) with $16 \mathrm{~S}$ survey data.

To infer differential richness, one first estimates richness for the specific communities of interest in each survey sample. The estimated values are then compared between sample groups with either fixed or mixed effects models, or with non-parametric statistical tests, possibly adjusting for sampling effort ${ }^{24-27}$. There are two types of sample-level estimates of richness. Observed richness refers to the number of taxa observed in a sample. Asymptotic richness is obtained by adding an estimate of the number of unobserved taxa to the number of observed taxa. Approaches to estimate asymptotic richness vary, but often assume that relatively uncommon taxa are the most informative ${ }^{28}$. Both types of richness estimates enable valid comparisons among macroecological communities ${ }^{24,25,28-30}$.

However, direct application of the aforementioned richness estimates and comparisons to $16 \mathrm{~S}$ microbiome data would ignore the plethora of uncommon and spurious taxa that inflate observed richness estimates in $16 \mathrm{~S}$ survey data ${ }^{27,31-34}$. When this artificial inflation in observed richness is ignored, we find that differential abundance of detected taxa confounds current methods for differential richness inference. The problem is severe when between-sample richness comparisons are made at lower taxonomic levels, e.g., genus. Thus, direct application of classical methods to microbiome differential richness inference is unreliable.

79 Attempts to overcome sequencing noise have been made. Chiu \& $\mathrm{ChaO}^{35}$, noting that singleton taxa are highly susceptible to sequencing noise, establish an improved estimator for undetected richness by relying on more abundant taxa (also see Willis ${ }^{36}$ ). However, the estimator is often numerically undefined at lower levels of the taxonomy, and still takes observed richness at face value.

84 Our results indicate that the observed frequencies of spurious taxa are determined by the output 85 abundances of input sequences, and thus need not be restricted to singleton frequencies alone. 
86 We therefore aimed to develop a flexible differential richness inference procedure for 16S

87 microbiome surveys - one that would not only allow investigators to seek sample-wide richness

88 changes across experimental groups (as is commonly done in modern metagenomics), but also

89 within genera or taxa collections of any particular interest, while accounting for false taxa

90 accumulations.

91 The paper is divided into several sections. Section 2.1, based on our own experiments and

92 exploratory data analyses guided by theory, presents multiple lines of evidence supporting the

93 view that most sub-genus taxa currently identified in $16 \mathrm{~S}$ surveys are spurious. This allows us to

94 exploit within-genus taxa accumulation data to derive a robust control for false taxa accumulations

95 (Methods section). Section 2.2 illustrates the confounded differential richness inferences arising

96 from current methods, when detected taxa exhibit a net non-zero relative abundance fold change

97 between sample-groups. Section 2.3 applies the proposed procedure (Prokounter) to a variety of

98 datasets and illustrates the value that differential richness inferences at lower taxonomic levels

99 add to clinical and public health related microbiome data analyses. For example, application of

100 Prokounter to a gut microbiome survey of a traveling individual ${ }^{2}$ identifies invading genera with

101 increased richness in member taxa, during and after an enteric infection. 


\section{Results}

\subsection{Most sub-genus taxa in $16 \mathrm{~S}$ surveys are likely technical artifacts}

104

105

106

107

108

109

110

111

$16 S$ surveys reconstruct target microbial populations by clustering sequencing reads. Spurious microbial taxa occur when the clustering procedure's error model fails to capture the entirety of sequence variation induced by the technical steps in $16 \mathrm{~S}$ sequencing. These steps include, but are not limited to, PCR amplification of $16 \mathrm{~S}$ material and sequencing (Fig. 1A).

To identify the major parameters underlying false taxa accumulations, we mathematically model the nucleotide substitution errors introduced by a chain of PCR amplification and sequencing processes allowing for back mutations (Supplementary Note 1). Under reasonable assumptions, we find that the rate of falsely classifying an error variant of a source sequence (type I error) using a priori fixed sequence similarity thresholds, strongly increases with the source sequence's recovered (i.e., output) abundance. The average recovered abundance is multiplicative in the source sequence's apparent input abundance and the total sampling depth (Supplementary Note 1). Thus, false sequence clustering decisions, and hence the resulting false clusters, increasingly accumulate with the true source sequence's recovered abundance, and not necessarily sample depth. We therefore identify a mechanism through which spurious clusters of sequences are increasingly identified as microbial taxa, regardless of the underlying biological reality.

Given the empirical observation that $16 \mathrm{~S}$ genetic segments are mostly limited in resolution to prokaryotic genera ${ }^{37-44}$, we explored within-genus taxa accumulations (i.e., the number of detected sub-genus taxa as a function of recovered genus abundances), in several publicly available $16 \mathrm{~S}$ surveys. In general, we expect genera to vary in their true richness and the relative abundances of member taxa. This must accordingly induce biological variation in the genusspecific taxa accumulation patterns. However, this expectation did not broadly hold in the several microbiome surveys analyzed here. Within-genus taxa accumulation patterns were highly concordant for several genera within study (Fig. 2A, Fig. S1-S3). Relative to the number of detected genera, which ranged from 60-400 across studies, a clustering analysis indicates that within-genus taxa accumulation data supports only 2-8 distinct accumulation patterns in each study (Table. S1). Multiple dominant genera can be clustered to the same accumulation pattern. In addition, relative to study specific covariates, a robust trend estimate of the within-genus taxa accumulation data explains the bulk of the variation in genus-specific and sample-wide taxa accumulations (Tables 1-2) in each study. Similar qualitative conclusions follow when genus recovered abundance is used as a predictor, instead of an estimated trend (Tables S2-S3). Finally, these qualitative and quantitative attributes of the accumulation patterns were obtained regardless of the $16 \mathrm{~S}$ clustering approach used (Tables 1-2). These results indicate a strong within-study regularity in observed taxa accumulations across genera and sample groups - as if most genera have similar taxa richness and evenness - suggesting a likely technical origin.

Single colony experiment To further verify these conclusions, we conducted a $16 \mathrm{~S}$ sequencing experiment on a target Pseudomonas aeruginosa population. The experimental sample was by itself overnight derived from a single $P$. aeruginosa colony (Supplementary Note 1). In a series of experimental samples, we varied both the input abundance of Pseudomonas cells and the PCR 
amplification cycles. Our mathematical model (Supplementary Note 1), which tracked the probability distribution of cell division induced nucleotide substitutions over generations, indicates that under no selection pressure, we can expect one biological 165 genotype in our input. An upper bound on the number of our input taxa is given by the number of $16 \mathrm{~S}$ genes generally found within the Pseudomonas genus $(\sim 4)$, times two for taxa clusters corresponding to forward and reverse complement strands. What we found was a rather different representation, rich with low abundant and poorly replicating taxa: the total numbers of observed Pseudomonas taxa were 1050 and 300 for clustering methods based on sequence similarity with respective thresholds of $99 \%$ and $97 \%$. The bulk of the newly identified Pseudomonas taxa preferentially contributed to the low frequency regime of the taxa abundance histogram (Fig. S4), suggesting that they are likely clusters of rare, erroneous $16 \mathrm{~S}$ sequencing reads generated during amplification and sequencing. Notably, taxa within-Pseudomonas, despite having a noisy occurrence with respect to amplification cycles and input cells (Fig. 3A), accumulated along the Pseudomonas genus recovered abundance axis in a clear, robust fashion (Fig. 3B). As expected, the stricter the sequence similarity threshold, the stronger the rate of taxa accumulations along the recovered abundance axis (Fig. 3B). Furthermore, taxa accumulations from several detected genera followed quantitatively similar patterns (Fig. 3C, Tables 1-2). From prior experiments in our laboratory and from control samples, we know Pseudomonas lab contaminants have very weak relative abundances. Restricting the above analysis to only those Pseudomonas taxa that track input cells, does not change the aforementioned conclusions qualitatively (Fig. S5).

Similar results on taxa accumulation patterns were also obtained for the multiple-genera Oral and Gut mock communities of the microbiome quality control project, handling lab $B\left(\mathrm{MBQC}^{27}\right)$.

Because true taxa are expected to replicate across study samples, we next explored sub-genus taxa occurrence rates (Fig. S19). In all studies, we find that over $50 \%$ of sub-genus taxa in over $50 \%$ of the detected genera did not replicate in more than $10 \%$ of the samples. Mock experimental communities are expected to represent a greater degree of homogeneity than real world communities as the latter may contain rare variants. Restricting analysis to experimental communities with single- and multiple- mock genera, we find that in eight out of nine datasets, over $50 \%$ of sub-genus taxa in over $50 \%$ of the mock genera replicated in less than $50 \%$ of the samples (Fig. S19). These results indicate poor within-study replicability of most sub-genus taxa.

Finally, because we expect true taxa richness and evenness to vary along the taxonomic tree, we explored taxa accumulations for the various taxonomic levels (i.e., family, order, class and phylum) in each study. Remarkably, the total number of observed taxa at any level of the taxonomic tree, was strongly predicted by recovered abundance alone and was not dependent on the taxonomic level considered (Fig. 2A, Fig. S20). These results indicate a strong regularity in taxa accumulations across taxonomic levels. 


\subsection{Spurious taxa confound differential richness inference}

181 That observed spurious sub-genus taxa increasingly accumulate with genus recovered 182 abundances leaves us with two expectations.

183 First, without appropriate corrections, inferring differences in a genus' number of associated taxa 184 (i.e., genus-wise differential richness) are highly likely to be confounded by the genus's respective difference in the recovered abundances (differential abundance). We observe that estimated genus-wise richness values from asymptotic estimators grew systematically with the genusspecific recovered abundances (Fig. S6). In addition to observed richness, estimates of unobserved richness can exhibit similar behavior (Fig. S7). This in turn induces an artifactual positive correlation between the resulting genus-wise differential richness fold changes and the genus-wise differential abundance fold changes (Fig. 2B, S8).

Second, inferring differential richness between sample-groups (i.e., sample-wide differential richness) are highly likely to be confounded by a net non-zero relative abundance fold change of detected genera. Straightforward simulations where spurious taxa are generated in an abundance dependent fashion illustrate this behavior (Fig. S6). Interestingly, illustrative examples of the same were rare in several $16 S$ surveys, suggesting that spurious taxa accumulations are comparable at the sample-level. Indeed, in many datasets, the relative abundance log fold changes of member genera were symmetric and concentrated around zero (Figs. S9-S10). Nevertheless, exceptions with asymmetric relative abundance log fold change distributions exist and a case in point is offered by the long-term time series study discussed below (Fig. S11).

200 In Supplementary note 2, we model the abundance dependent generation of spurious taxa in 16S 201 surveys within the sample theoretic framework of $\mathrm{Chao}^{29}$ and Harris ${ }^{45}$ and find that the above observations agree with theory.

\subsection{Prokounter enables flexible differential richness inference}

To overcome the aforementioned biases when applying current richness estimators to 16S surveys and to establish a flexible differential richness inference approach, we developed Prokounter and applied it to several microbial communities including those from a long-term time series study, hydrocephalus cohort, waste-water treatment plant and our pseudomonas dilution experiment.

While zero-truncated statistical models offer one route to modeling member inclusions in a population survey, the same can be achieved by incorporating appropriate predictors in a regression context ${ }^{46}$. The former is the approach taken by some classical richness estimators to model species abundance ${ }^{28,47}$. We take the latter view and proceed as follows. Based on the results from section 2.1, we assume that most sub-genus taxa in $16 \mathrm{~S}$ surveys are false. This allows us to exploit a $16 \mathrm{~S}$ survey's overall sub-genus taxa accumulation trend, along with any systematic genus-specific effects, as a sampling effort dependent control for false taxa accumulation (Methods). This control is exploited within standard regression methods for differential richness inference. 
With a few 165 surveys, we illustrate the insights offered by the proposed procedure, Prokounter, in achieving genus-specific and sample-wide differential richness inferences.

Unlike other estimators analyzed here $\left(\mathrm{Chao}^{29}, \mathrm{ACE}^{48}\right.$ and Breakaway $\left.{ }^{49}\right)$, the uncorrelatedness of Prokounter's richness statistics with genus-wide differential abundance statistics is clear in each dataset (Fig. 2B,C, Fig. S12). Breakaway's estimates were the most variable, often accompanied by wide confidence intervals. On several occasions, genus-specific differential richness estimates were not well defined in numerical value when using current richness estimators for numerical, and not necessarily statistical identifiability reasons. Sample-wide inferences agreed among all methods in most cases, except when detected genera exhibited a net non-zero relative abundance fold change distribution.

In all surveys below, asymptotic genus-wise and sample-wide richness estimates heavily tracked their respective observed richness values (97-100\% Pearson correlations, Figs. S13-S17).

Long-term time series study Based on a clustering analysis of abundance profiles, David et al., ${ }^{2}$ identified that a distinct sub-group of the phyla Firmicutes replaced another Firmicutes sub-group, post-enteric infection, in the gut microbiome of an individual relocating to a different country. Prokounter refines this result further by identifying several Firmicutes genera (Faecalibacterium, [Ruminococcus], Oscillospora) that are less rich post-infection. On the other hand, Dorea and Coprobacillus, members of Firmicutes, were found to have significantly increased richness in infection and post-infection samples. The genus Acinetobacter from the phylum Tenericutes was found to have significantly higher richness in samples collected during infection, while this was not the case post-infection. Thus, differential richness adds another state variable to the microbiome state specifications of the original study.

In David et al's dataset, sample-wide inferences disagreed among the methods compared. Prokounter produced negative richness inferences for both infection and post-infection samples consistent with antibiotic exposure. Chao1/Betta and ACE/Betta indicated reduced richness postinfection with a relatively weak significance for reduced richness in infection samples. Breakaway/Betta failed to reject any of the corresponding null hypotheses ( $p$-value $=0.99$ infection and $p$-value $=0.74$ post-infection), potentially owing to the very high variability of Breakaway estimates. As established in the previous subsection, these differences in inferences likely stem from the asymmetric differential abundance of detected genera in the samples collected during infection.

Pathogenesis We applied Prokounter to a $16 \mathrm{~S}$ survey of the cerebrospinal fluid from hydrocephalus children hypothesized to have infectious $(\mathrm{PIH})$ and non-infectious (NPIH) origins ${ }^{50}$. 251 We intuitively expected, and observed, that the cerebrospinal fluid enveloping the central nervous 252 system to register lower richness compared to laboratory controls. PIH samples had relatively lower richness compared to clinical control samples.

254 A genus that is positively differentially abundant, along with a negative differential richness 255 estimate might indicate invasion of a sub-species. Genus-specific differential richness inference 256 with Prokounter yields two genera as having lowered richness in the PIH samples: Paenibacillus 
and Streptococcus. Paenibacillus was the dominant pathogenic genus identified with the PIH phenotype using $16 \mathrm{~S}$ data ${ }^{50}$.

Waste-water treatment To demonstrate an ecological monitoring application, we applied Prokounter to $16 \mathrm{~S}$ data arising from a waste-water treatment plant ${ }^{51}$. The method indicates that relative to the effluent, sample groups from each of the post-treatment stages have significant negative microbial richnesses. These results readily agree with our expectation of a publicly implemented waste water treatment protocol. Chao1/Betta, ACE/Betta produced similar results. Breakaway/Betta failed to reject the null for sample groups corresponding to effluent $(p=.065)$ and inlet to pumphouse $(p=.692)$.

Using differential abundance analysis, the original study highlighted the persistence of Legionella and Mycobacterium in post-treatment samples calling into question the efficacy of the treatment process. Performing genus-specific differential richness analysis with Prokounter indicates that the treatment plant reduces the richness associated with several types including Mycobacterium. We did not detect Legionella as reduced in richness in the effluent. These results indicate that waste-water treatment has been effective with removing Mycobacterium sub-types.

Pseudomonas dilution study The Pseudomonas dilution experiment varied two parameters of a $16 S$ experimental pipeline: amplification cycles, and input cells of a single colony derived 274 microbial population.

275 Increased amplification cycles can allow increased sampling of both contaminant and input genera. Thus, within further sampling constraints imposed by the multiplexed nature of the experiment, we expect sample-wide richness to grow with amplification cycles. Sample-wide differential richness inference from all methods matched this expectation.

It is well known that the abundance of lab contaminants falls with input loads [34]. If the dynamic range in input loads is sufficiently high, we can expect inferred sample-wide richness to fall with input Pseudomonas cells. Results from Prokounter, Chao1/Betta and ACE/Betta matched this expectation. Breakaway/Betta failed to reject the corresponding null hypotheses $(p=0.4)$.

The genus of principal interest in this experiment is Pseudomonas. The genus-wide differential richness results from Prokounter indicated a decrease in richness with respect to input cells and an increase with respect to amplification cycles. This is in line with our expectations as we expect the detection rate of lab contaminant Pseudomonas species to grow with amplification cycles, and fall with input Pseudomonas loads. In direct contrast, Chao1/Betta and ACE/Betta, confounded by input Pseudomonas's increasing abundance, indicated a Pseudomonas richness increase with input cells ( $p=0$ for both), and Breakaway/Betta failed to reject $(p=0.251)$. 


\section{Discussion}

Summary: $16 \mathrm{~S}$ microbiome surveys reconstruct target microbial populations by clustering sequencing reads. Spurious microbial taxa occur when the clustering procedure's error model fails to capture the entirety of sequence variation induced by the technical steps in $16 \mathrm{~S}$ sequencing (Supplementary Note 1, Fig. 1A). We have shown that the false taxa thus generated not only inflates the estimate of a (microbial) community's richness (Supplementary Note 2, Fig. S6), but they also cause taxa differential abundance to confound differential richness inferences (Fig. 2B, S8). This occurs because every false taxon is generated through errors from one or a few true (i.e., input) taxa, and hence, their rates of production increase with the output abundance of the corresponding source taxa (Supplementary Note 1). Based on our result that most subgenus discoveries are likely false (Section 2.1), we have established abundance dependent controls for false taxa accumulations using a given survey's within-genus taxa accumulation data (Methods, Fig.2C, S2, S18). We have shown that our strategy overcomes the confounding problem (Fig. 2C, S12). And we have illustrated the utility of differential richness inferences in individual and public health related microbiome data analyses (Section 2.3).

Assumption: Our approach assumes that most sub-genus taxa in 16S surveys are spurious and are poor representatives of the underlying microbial community. We have provided several lines of evidence to support this conclusion: First, our mock experiment of an overnight derived microbial population indicated that observed richness can be severely inflated (Fig. 3, S5). Our expectation was set in part by a mathematical model of cellular reproduction, where we tracked the probability distribution over substitutions, over generations (Supplementary Note 1). Second, in a manner similar to what we would expect of low probability errors, most sub-genus taxa in both controlled mock and real world datasets are rare and show poor replicability across samples (Fig. S19). Third, within-genus taxa accumulation patterns in several publicly available datasets, including those from single- and multi-genera mock experiments, appear remarkably regular as if most genera in $16 \mathrm{~S}$ surveys have similar richness and taxa evenness (Fig. 2-3, S1-S3, Tables 12, S2-S3). Fourth, the total number of taxa observed for any taxonomic level was strongly determined by the category's recovered abundance alone and was not dependent on the level itself (Fig. 2, S20). Finally, the literature offers abundant support for abundance dependent false taxa generation in $16 \mathrm{~S}$ surveys, of which we note a closely related few. Kunin et al., ${ }^{32}$ demonstrate the large number of false Escherichia taxa that arise in a $16 S$ survey of a target E.coli population (also see Degnan and Ochman ${ }^{52}$, Pinto and Raskin ${ }^{53}$ ). Based on the empirical observation that the number of false taxa generated are sampling effort dependent, Schloss et al., ${ }^{54}$ recommend that community-level comparisons be made at comparable sampling depths. Haas et al., ${ }^{55}$ illustrate the predictable, abundance dependent generation of false chimeric taxa within genera in mock communities.

Implications for richness theory and automated ecological surveys: False microbial taxa in $16 S$ surveys arise because automated procedures to reconstruct taxa misclassify sequencing reads from their true types. In Supplementary Note 1, we analyzed the influence of amplification and sequencing induced substitutions in causing misclassifications (also see Schloss ${ }^{56}$, and Sze and Schloss ${ }^{57}$ ). In Supplementary Note 2, we mathematically modeled the false taxa that arise 
through misclassification and showed in part that a traditional asymptotic richness estimator $\left(\mathrm{ChaO}^{29}\right)$ is biased under this more general sampling scenario. The severity of bias is determined by sampling parameters. Together with the results mentioned in the previous paragraphs, we conclude that classical richness theory, which predominantly focuses on estimating undetected richness while assuming observed richness at face value, should be generalized for observed species misclassifications in modern high throughput and highly automated surveys.

Asymptotic richness estimators track observed richness values in 165 surveys: In the several 16S surveys considered here, asymptotic richness estimates tracked observed richness values both sample-wide and at within-genera levels (Fig. S13-S17). Our mathematical models and simulations that incorporate false taxa accumulations within the sampling theoretic framework of $\mathrm{ChaO}^{29}$ and Harris ${ }^{45}$ indicate that such tracking can arise when the apparent richness (i.e, the true plus false richness) and not necessarily true richness, is undersampled in a survey (Supplementary Note 2). This explains the observed tracking in the Pseudomonas genus in the Pseudomonas dilution experiment, where we do not expect undersampling of the true Pseudomonas community (Fig. S13).

False discovery control in differential richness analysis, confounding with differential abundance: Hughes et al., ${ }^{58}$ argue that traditional macroecological richness estimators continue to enable robust sample-wide richness comparisons in $16 \mathrm{~S}$ surveys. Our analysis identifies exceptions (Section 2.3, Long-term time series study) and clarifies the practical conditions under which controlling for spurious discoveries become important. In particular, we find that false taxa accumulations cause abundance dependent inflation in observed taxa numbers and their frequencies (Supplementary Note 1,2), causing differential (relative) abundances of detected taxa to confound differential richness inference with traditional methods (Fig. 2B, S6-S8, S11). When spurious taxa accumulations are comparable across contrasted experimental groups, no such confounding arises (Fig. S9-S10). Our empirical analyses indicate that such an assumption is too strong for making differential richness inferences at lower taxonomic levels (e.g., genusspecific) of a microbial assemblage (Fig. 2B).

Relaxing microbiome richness comparisons to taxonomic groups: Microbiome analyses frequently restrict richness comparisons to the entire microbial assemblage obtained in study samples (sample-wide richness inference). From the perspective of deriving health and ecological indicators based on community assemblages, analysis of a community's finer organization levels is equally interesting $2,8,10-12,17$. Our genus-wise differential richness results (Section 2.3) indicate that contrasting richness for taxonomic sub-groups can enable practically useful inferences and add interesting dimensions to microbiome state space descriptions.

Within-genus taxa accumulation structure and the trend estimator: Our results document reliable across-genera regularity in the patterns of within-genus taxa accumulations, across many studies and genus-specific experiments (Fig. 2-3, S1-S3, Tables 1-2, S2-S3). We speculate that genus abundances, in contrast to sampling depth, more accurately track the sampling rate of false sequence variation in $16 \mathrm{~S}$ surveys for at least two reasons. First, commonly exploited 16S rRNA target segments are limited in resolution beyond genus level ${ }^{37-44}$. Second, genus recovered 
abundances, unlike total sampling depth, normalize for the sampling rates of distinct genera. This restricts us from mixing taxa accumulation statistics over truly disparate input biological sequences from distinct genera, while allowing us to preserve any systematic genus specific effects. We used a robust trend estimate of the within-genus taxa accumulation data to model spurious taxa accumulation (Methods, Fig. 2-3, S1-S3). The coherent accumulation of a large number of detected taxa translated to low estimation uncertainties. These curves were not necessarily linear in the recovered genus abundances (Fig. S1-S3). The systematic genusspecific contributions to this trend can arise due to between-genera variation in both detectable true input sequence diversity (copy number ${ }^{43}$ or number of distinct cell types) and $16 \mathrm{~S}$ sequencing noise ${ }^{56,57}$.

Abundance dependent control in bioinformatic sequence analysis: Beyond differential richness inference, there is a need for recovered abundance dependent control in other (meta)genomic sequence analyses e.g., sequencing read mapping and taxonomic annotation, which exploit fixed sequence similarity thresholds. Probabilistic methods have a natural incorporation of abundance in clustering/mapping decisions. In all cases however, poor error models would continue to drive false taxa accumulations. It must be noted that we have not analyzed false negative rates in this study ${ }^{59,60}$.

Limitations of differential richness inference Observed (and reportedly, asymptotic ${ }^{61}$ ) richness estimates cannot forecast crossing over of species accumulation curves that can in principle occur with additional sampling effort. However, differential analysis of both these estimates over realized sampling effort is still useful for detecting perturbations to the evenness of a biological community ${ }^{58,62}$, and is thus effective for deriving predictors of individual and environmental health.

Future work. There are several avenues for future research. First, an integrated estimation procedure of false taxa accumulation rates and differential richness fold changes would lead to more appropriate p-values under the assumed statistical models. Second, development of ecological richness estimators in the presence of species misclassifications would be a valuable addition to the literature. Supplementary Note 2 considers a simple but a useful special case. Third, $16 \mathrm{~S}$ surveys on mixtures of microbial species with varied relatedness and controlled input richness levels, would enable a joint characterization of detectable $16 \mathrm{~S}$ resolution, taxa reconstruction algorithms and richness estimators. Fourth, control for multiple testing over tree structured hypotheses can be incorporated if one wishes to automate hypothesis testing over taxa collections defined by subtrees of a taxonomic tree ${ }^{63,64}$. Finally, all our empirical observations were based on a set of $16 \mathrm{~S}$ surveys that operate over partial $16 \mathrm{~S}$ gene targets. Because full length $16 S$ surveys also involve amplification, and sequencing protocols ${ }^{41}$, we expect the qualitative nature of our results to generalize to such surveys, perhaps at a lower taxonomic level (e.g., species), and this can be explored.

Taken together, this paper significantly clarifies the dynamics of spurious discovery accumulation in 16S surveys, presents strategies for modeling their generation, demonstrates the need to control for the observed false discoveries in microecological surveys while deriving differential richness inferences, and offers a flexible practical solution to achieve the same. 


\section{Methods}

\section{Prokounter}

424 Our proposed procedure for differential richness inference works in two steps. A control for false 425 taxa accumulation is established first. The estimated control is subsequently exploited within 426 standard generalized linear models for differential richness inference.

427 Let $n_{g j}$ denote the reconstructed number of taxa for genus $g$ in sample $j, y_{g j}$ denote the corresponding recovered abundance (i.e., genus's total count in the sample), and $\tau$ represent the sample depth.

430 Let $f_{t}\left(\log y_{g j}\right)$ indicate the logged technical contribution to taxa accumulation for a given genus and its recovered abundance level. This function is used to model the log of the expected false taxa accumulation. Its estimate $\hat{f}_{t}\left(\log y_{g j}\right)$ is obtained using within-genus taxa accumulation data as follows.

Estimating the technical contribution $\widehat{f}_{t_{-}}$We explored two strategies to estimate a robust within-genus accumulation trend.

A semi-parameteric smoothing spline model is assumed on $z_{g j}=\log n_{g j}$,

$z_{g j} \mid g, y_{g j}=\eta\left(g, y_{g j}\right)+\varepsilon_{g j}=\kappa+f_{R}\left(\log y_{g j}\right)+f_{G}(g)+f_{G R}\left(g, \log y_{g j}\right)+\varepsilon_{g j}$

with $\varepsilon_{g j} \sim N\left(0, \sigma^{2}\right)$, and appropriate side conditions are placed on $f(\cdot)$ (Chapters 2-3 $\left.{ }^{65}\right)$. Here $\kappa$ and $f_{R}(\cdot)$ denote the intercept and recovered abundance dependent components; $f_{G}$ and $f_{G R}$ indicate the genus and its respective interaction functions with the recovered genus abundance.

442 Briefly, $\eta$ is estimated as a unique solution to the penalized optimization problem: $\hat{\eta}=$

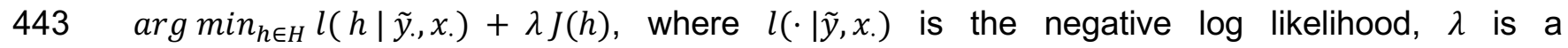
444 regularization parameter and $J(\cdot)$ is a roughness penalty that penalizes overfitting of $h$ to the data. 445 The specification of $J(\cdot)$ involves, in part, integrals of squared second order derivatives of the 446 estimand over the range of $\log y_{g j}$, thereby enforcing smoothness. Supplementary Note 4 offers 447 more details on the model construction and an exact correspondence to example 2.7 in $\mathrm{Gu}^{81}$. 448 Numerical optimization is performed using the R package $g s s^{65}$. Supplementary figures S2 and 449 S18 offer examples of the fits that result.

450 The technical contribution to taxa growth is estimated as $\hat{f}_{t}\left(g, \log y_{g j}\right)=\kappa+\hat{f}_{R}\left(\log y_{g j}\right)+\hat{f}_{G}(g)$. 451 Only the significant genus effects are retained after multiple testing correction with the Benjamini452 Hochberg procedure. When the genera contributions are null or similar, as we observed 453 empirically in several datasets (e.g., Fig. S9, S10), $\hat{f}_{t}\left(g, \log y_{g j}\right) \propto \hat{f}_{R}\left(\log y_{g j}\right)$.

454 The latter observation inspires the following alternative strategy: estimate $\hat{f}_{t}(\cdot)$ as a net average 
within-genus accumulation curve using the loess smoother. Both options are made available in our software. As expected, inferences arising and the results in tables 1-2 are similar with both approaches. Fig. S3 offers examples of the fitted trends. The spline strategy does offer better control in the presence of systematic genus effects (Fig. S18).

459 For consistency, in this paper, we have chosen the spline strategy.

460 The fitted $\widehat{\boldsymbol{f}}_{\boldsymbol{t}}$ can be used to control for false taxa accumulation in standard differential richness inference procedures. In Prokounter, we incorporate it through the models presented below.

462 Differential richness inference We use Greek letters to indicate regression parameters. A -in the subscript indicates vectorizing over the subscript. $X$ denotes the experimental design matrix. Genus-specific, sample-wide and taxa collection models are presented in equations (2)-(4) below. In each case, given the quantity modeled, reasonable transformations of the estimated logged technical contribution, $\widehat{\boldsymbol{f}}_{t}$, based on eqn. (1), are used. Terms involving $X$ below can be viewed to approximate the effects arising from genus-recovered abundance interaction terms 468 from eqn. (1).

Genus-specific differential richness inference the conditional mean of the observed richness is modeled through the link:

$471 \log E\left[n_{g j} \mid y_{g}, X, f_{t}(\cdot)\right]=X_{j}^{T} \mu_{g}+v_{g} f_{t}\left(\log y_{g j}\right)$

where the right hand side is an approximate form for the log of the conditional expectation of the 473 right hand side of eqn. (1).

474 Sample-wide differential richness inference For inference across sample groups, we posit:

$\log E\left[n_{+j} \mid y_{g}, X, f_{t}(\cdot)\right]=X_{j}^{T} \zeta+\gamma \log \sum_{g: y_{g j}>0} e^{f_{t}\left(\log y_{g j}\right)}$

where the + indicates summation over a subscript. As in eqn.(2) the right hand side of eqn.(3) is an approximate form for the log of the conditional expectation of the right hand side of eqn.(1), now summed over $g$. The net sample-wise technical contribution is modeled as a simple sum of the technical contributions from the genera detected in the sample. Although eqn.(3) does not immediately arise from eqn.(2), we find the simplicity and emphasis on dominant contributors to the sum, the more abundant genera, appealing. In addition, we often find that $v_{g} \approx 1$ and $\gamma \approx 1$ in

483 Differential richness inference for arbitrary collections of genera For an arbitrary taxonomic group $484 k$ (e.g., phyla), with a set of member genera $G_{k}$, we assume : 
only to those genera considered within the collection.

489 Keeping to the traditional theme of continuous Poisson mixtures driving sample-wide species accumulations, we chose Negative Binomial variance functions when performing sample-wide inferences, and Poisson variance functions for genus-specific richness inferences. For the several studies considered here, the estimated overdispersion coefficients for sample-wide Negative Binomial models were in the range of $10^{-3}$ to $10^{-1}$. For well expressed genera, inferences and model diagnostics were not sensitive to the two distribution assumptions. Parameter estimation and inference on the regression parameters $\mu_{g}$, $\zeta$ and $\psi_{k}$ were performed using R's glm function. Maximum likelihood estimation with iteratively reweighted least squares converges rapidly in about ten iterations or less. Speaking to the explanatory power of $\widehat{f}_{t}$, as implied by tables $1-2$, the residual deviance is often small, on the order of the residual degrees of freedom. To gauge reproducibility of inferences over fitted $\hat{f}_{t}(\cdot)$, confidence intervals based on the bootstrap $t^{66}$ are also available for the regression coefficients of the sample-wide differential richness inference

501 model.

The above models, which were used to generate the results in the applications section, exploit observed richness as response variables and are therefore non-asymptotic in nature. In the several 16S surveys considered here, asymptotic genus-wise and sample-wide richness estimates heavily tracked their respective observed richness values $(97-100 \%$ Pearson correlations, Figs. S13-S17). We therefore propose the same regression models above for standard inverse variance weighted regression analyses of asymptotic richness estimates. As expected, results from such a procedure were similar to those obtained with observed richness as the response variable. Also see reference ${ }^{26}$ for a heterogeneity test of potential interest.

We implement these procedures in an R package Prokounter. Supplementary Note 2 presents further discussions on the regression models above.

\section{Package and code availability:}

513 The R package Prokounter is available from the link: https://github.com/mskb01/prokounter

514 Code for the paper is available from the link : https://github.com/mskb01/prokounterPaper

Richness estimators and differential analyses: Estimates and standard errors for Chao 1 and ACE estimators were calculated using the R package vegan ${ }^{67}$. Breakaway estimates and standard errors were obtained using the $R$ package Breakaway. Differential richness inferences corresponding to the three estimators were obtained with the $R$ package $B \operatorname{Btta}^{26}$. Rarefaction based interpolated and extrapolated richness estimates and standard errors were obtained using the package $i N e x t^{68}$. The R package doParalle $\left.\right|^{69}$ was used for several parallel computing tasks. sample-wide and genus-specific differential analyses reported in the applications section.
1. Hydrocephalus ${ }^{50}$ (PIH100 FST97) - Control and Case. 
2. Wastewater ${ }^{51}$ (WW FST99) - Influent, Effluent, Before UV treatment, After UV treatment, Pond storage, and Inlet to pumphouse for subsequent spray irrigation.

3. MBQC, Handling lab B (MBQC-HLB) - Gut mock, Oral mock, the rest of the stool samples were typed as Other.

4. Time series study ${ }^{2}$ (TS FST97, Donor B) - based on the original study, three time windows were established to define sample groups: days up to to 150 were categorized as preinfection, days from 151 upto 159 as infection, and days post 159 were typed as postinfection.

535 Dilution experiment: A monoisolate was prepared overnight from a Luria-Bertani (LB) agar cells in phosphate buffer saline (PBS) was generated. DNA was isolated, $16 \mathrm{~S}$ amplified and sequencing libraries were prepared as previously described ${ }^{50}$. Briefly, DNA was isolated using the Zymobiomics DNA miniprep kit following manufacturers protocol with bead beating and proteinase $\mathrm{K}$ treatment. For $16 \mathrm{~S}$ amplification, primer-extension polymerase chain reaction (PE$\mathrm{PCR}$ ) of the $\mathrm{V} 1-\mathrm{V} 2$ region was performed using an $\mathrm{M} 13$ tagged 336R universal primer as previously described ${ }^{70}$ and amplification cycles were varied. Briefly, target DNA was mixed with a $10 \mu$ of $10 \mathrm{X}$ buffer, and annealed with M13 tagged $336 \mathrm{R}$ by first heating to $95^{\circ} \mathrm{C}$ and then cooling to $40^{\circ} \mathrm{C}$ slowly. The annealed product was extended using Klenow polymerase $(5 \mathrm{U} / \mu \mathrm{l}$ and primers digested with 20U/ul Exo I (NEB, USA), then amplified with 500 nM primers (805R and M13) using the MolTaq 16S Mastermix (Molzym GmbH \& Co Kg, Germany). Library preparation was done with the Hyper Prep Kit (KAPA Biosystems, USA) following the manufacturer's protocol and libraries were sequenced on MiSeq using the 600 cycle v3 kit.

$16 S$ datasets and taxa reconstruction pipelines: The mouse microbiome $16 \mathrm{~S}$ data was obtained from the R/Bioconductor package metagenomeSeq ${ }^{71}$. The moderate to severe diarrheal $16 \mathrm{~S}$ survey was obtained from the R/Bioconductor package $m s d 16 S^{72}$. The long-term time series $16 S$ survey $^{2}$ was obtained from the supplementary data of the corresponding paper. The wastewater $16 \mathrm{~S}$ survey ${ }^{51}$ was obtained on request from the authors of the original study. MBQC handling laboratory B's (HL-B) sequencing reads was obtained from the Microbiome Quality Control (MBQC) project ${ }^{27}$.

We generated three varieties of taxa count data from each of the Pseudomonas, PIH100 16S and MBQC HL-B (handling lab B) sequencing data. These include sequence similarity threshold based taxa clustering methods for $99 \%$ and $97 \%$ sequence similarities (Qiime1), and a probabilistic taxa clustering method (Dada2) as follows. (v0.38) to remove universal adapters and low-quality reads. Reads with ambiguous bases were removed or truncated using Dada2's filterAndTrim ${ }^{74}$ function. The 16S V1-V2 regions in both our 
reverse reads for the Pseudomonas dataset. These numbers were respectively $200 \mathrm{bp}$ and $190 \mathrm{bp}$ for PIH100. For HL-B, we removed the first $2 \mathrm{bp}$ following the primers in the forward and reverse reads. This allowed us to neglect the trailing low quality bases adversely affecting the taxa reconstructions, while still allowing for sufficient overlap to merge paired-end reads.

572 Reads with either the designed primers or their reverse complements were filtered using 573 cutadapt $^{75}$. The quality filtered reads were then clustered with Qiime $1^{76}$ and Dada2 ${ }^{74}$ as below.

575 Qiime 1 : Quality filtered forward and reverse reads were merged using $P$ ear ${ }^{77}$, and then clustered using pick_open_reference_otus.py (Qiime1 version 1.9.1), which implements the Qiime1 open reference OTU clustering algorithm. Briefly, closed reference clustering of merged reads were performed against the Silva132 database at $97 \%$ and $99 \%$ sequence similarity thresholds, using Uclust $^{78}$ v.1.2.22q. Reads that did not map to the database were subsampled and used as new centroids for a de novo OTU clustering step at the respective sequence similarity thresholds. Remaining unmapped reads were subsequently close clustered against the de novo OTUs. Finally, another step of de novo clustering was performed on the remaining unmapped reads. Taxonomy was assigned to taxa representative sequences with Uclust based on the Silva132 ${ }^{79}$ database. These sequences were filtered with Pynast $^{80}$, and OTU tables generated.

Dada2: Dada2 allows denoising forward and the reverse reads independently. Error rates were estimated separately for the quality filtered forward and reverse reads for each sample. This estimation step is based on a sample of reads for computational tractability. Reads were deduplicated and sequence clusters inferred based on the estimated error rates. Taxa from forward and reverse reads were merged at the end of the workflow. Chimeric taxa were removed with the function removeChimeraDenovo. The resulting taxa were assigned taxonomic labels based on the Silva132 database, using their naïve Bayes classifier. 
bioRxiv preprint doi: https://doi.org/10.1101/2021.11.07.467583; this version posted December 1, 2021. The copyright holder for this preprint

(which was not certified by peer review) is the author/funder, who has granted bioRxiv a license to display the preprint in perpetuity. It is made available under aCC-BY 4.0 International license.

\section{Acknowledgements}

594 This project was supported by an NIH Director's Transformative Award 1R01Al145057. We thank

595 the Genome Science Facility at the Penn State University College of Medicine for performing the 596 sequencing for the Pseudomonas dilution study. 


\section{Figures}

A

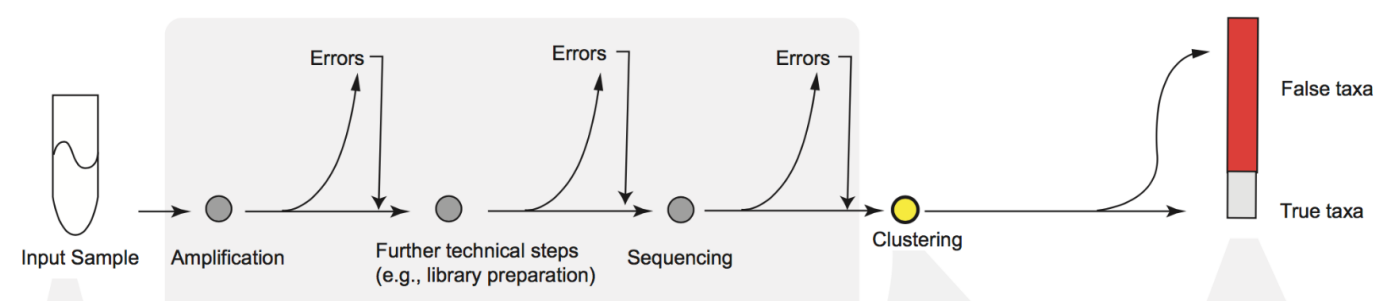

B

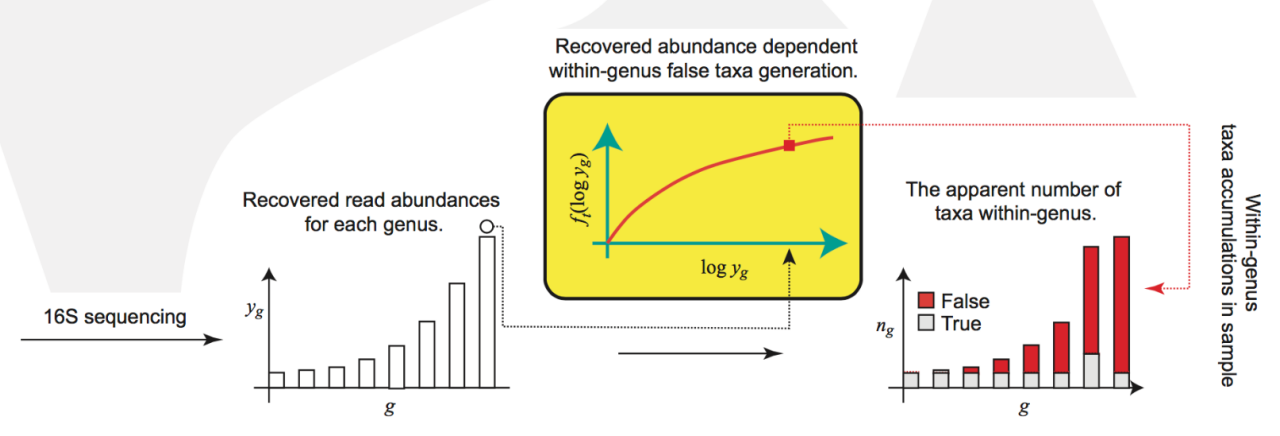

Figure 1. Within-genus false taxa accumulation structure. (A) Sequences in input samples are subjected to various technical steps during $16 \mathrm{~S}$ sequencing (gray shade). The output reads from 165 sequencing are clustered for sequence similarity using a methodology of choice. Of the number of taxa (clusters) thus reconstructed, some are true, i.e., equal in sequence to those in the input sample, the rest are spurious i.e., false (red). (B) For every genus, the accumulation is determined as a function of its recovered abundances. Notation: $n_{g}{ }^{0}$ the respective true number of taxa associated (true richness), $y_{g}$ the genus recovered abundance, $f_{t}(\cdot)$ the abundance dependent technical component driving false taxa accumulations within-genus. 
A

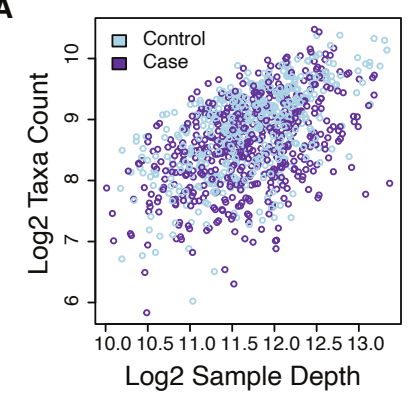

B

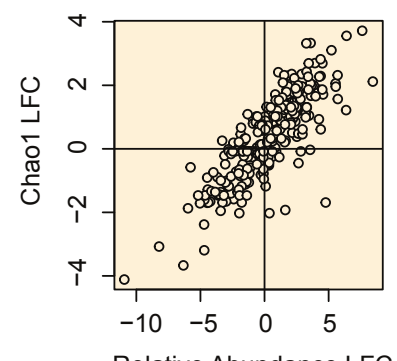

C

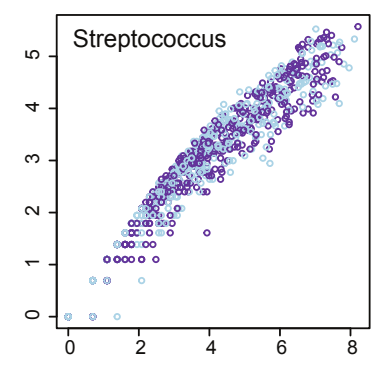

Log2 Recovered Streptococcus Abundance

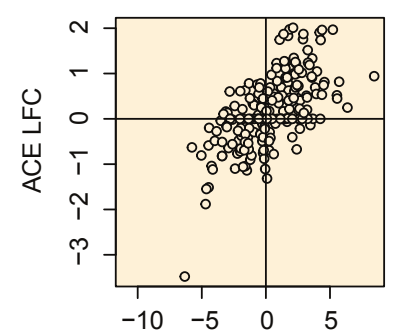

Relative Abundance LFC

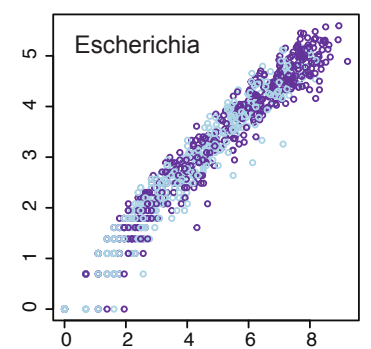

Log2 Recovered Escherichia Abundance

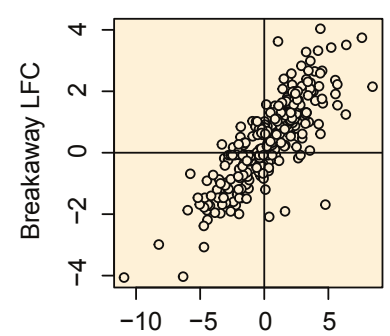

Relative Abundance LFC

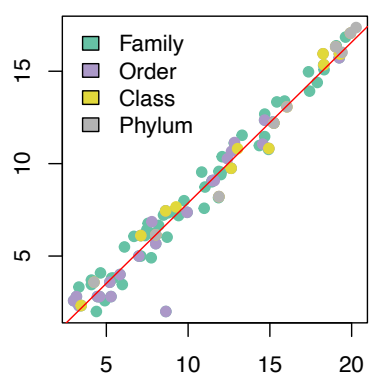

Log2 Recovered Abundance
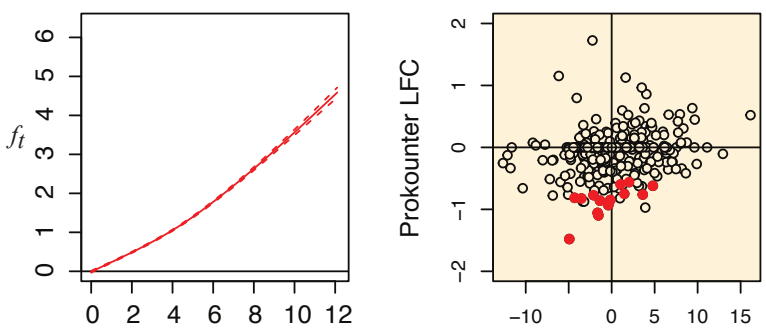

Log2 Recovered Abundance

Relative Abundance LFC

Figure 2. Concordant taxa accumulations across genera, confounded differential richness inference and the Prokounter strategy. (A) Sample-wide taxa accumulations are visualized with respect to sample depth (left). Within-genus taxa accumulations are visualized with respect to the total recovered genus abundances for two genera, i.e., the sum of the abundances of all taxa within the genus (center). Dataset-wide taxa accumulations for any taxonomic level is strongly predicted by recovered abundance alone (right). Red line illustrates a linear fit. (B) Differential richness log-fold changes (LFC, $y$-axis) track differential relative abundance fold changes (LFC, x-axis) in the waste-water treatment survey. (C) Prokounter exploits within-genus accumulation data to model false taxa accumulation rates. When exploited in a standard Poisson regression setting, the resulting differential richness fold changes are uncorrelated with genuswide differential abundance statistics (right). Dashed lines represent confidence intervals. Points survey. 
A

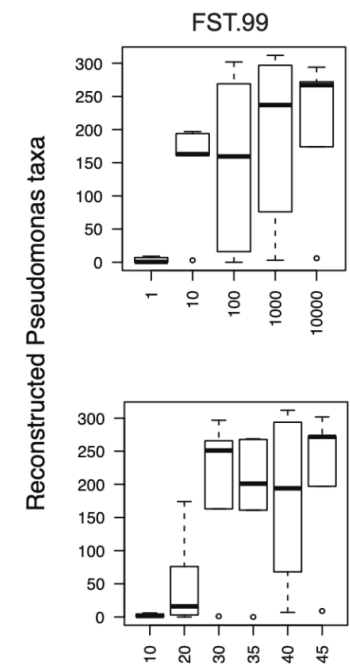

c

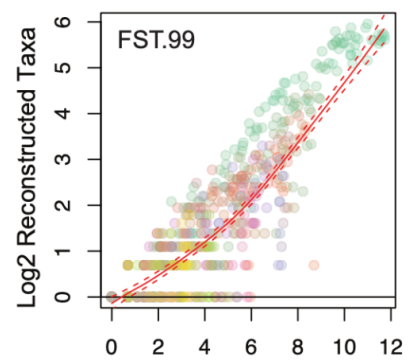

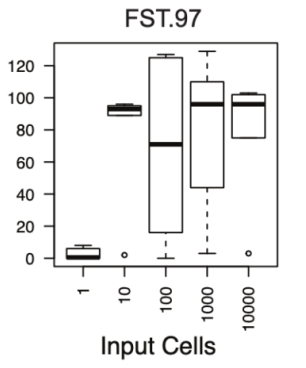
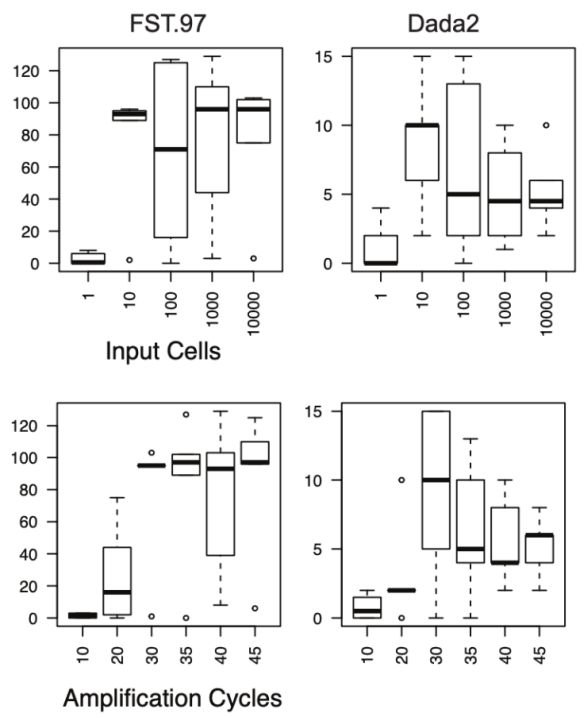

B

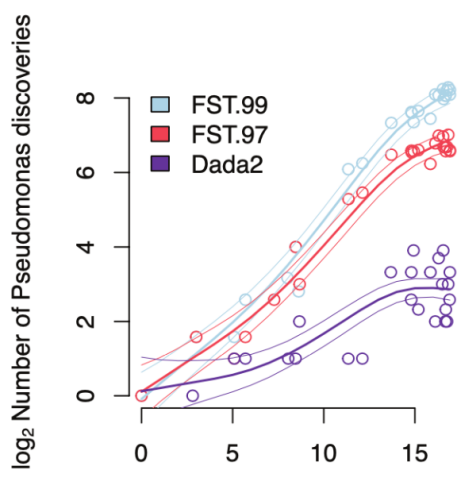

$\log _{2}$ Recovered Pseudomonas Abundance

Figure 3. False microbial discoveries accumulate along the recovered abundance axis in the Pseudomonas dilution study. (A) For each taxa clustering method, the observed variation in within-genus Pseudomonas taxa accumulations are driven by experimental and technical parameters. Contaminant Pseudomonas are expected to fall with input loads, indicating false discovery accumulations at higher recovered Pseudomonas abundances. (B) The genus recovered abundance axis offers a succinct representation for taxa accumulations. Average and the $95 \%$ point-wise confidence intervals for the logged within-Pseudomonas taxa accumulation trends are shown with colored lines for each method, with colored circles indicating the respective observations. (C) An overlay of taxa accumulations across multiple detected genera in the study. Colors indicate genera. 
Tables

\begin{tabular}{|l|l|l|l|l|l|l|}
\hline Dataset & Year & 16S segment, Sequencing\& Clustering & Pseudo $R_{\text {trend }}^{2}$ & Pseudo $R_{\text {trend }+ \text { design }}^{2}$ & AIC trend & $A I C_{\text {trend }+ \text { design }}$ \\
\hline Mouse [59, 60] & 2009 & V2, 454, FST.97 & $96.82 \%$ & $98.68 \%$ & $1.250 \times 10^{4}$ & $1.0273 \times 10^{4}$ \\
Diarrhea [1] & 2014 & V12, 454, FST.99 & $98.52 \%$ & $98.96 \%$ & $1.2323 \times 10^{5}$ & $1.1455 \times 10^{5}$ \\
Time series [2] & 2014 & V4, GAIIx, FST.97 & $94.43 \%$ & $98.46 \%$ & $3.9401 \times 10^{4}$ & $3.0813 \times 10^{4}$ \\
Wastewater [48] & 2018 & V34, MiSeq, FST.99 & $91.70 \%$ & $95.51 \%$ & $3.7400 \times 10^{4}$ & $2.3682 \times 10^{4}$ \\
MBQC-HLB $^{(97)}[27]$ & 2017 & V4, MiSeq, FST.97 & $97.15 \%$ & $98.66 \%$ & $1.0710 \times 10^{5}$ & $9.2275 \times 10^{4}$ \\
MBQC-HLB $^{(99)}[27]$ & 2017 & V4, MiSeq, FST.99 & $98.67 \%$ & $99.29 \%$ & $1.089 \times 10^{5}$ & $9.6674 \times 10^{4}$ \\
MBQC-HLB $^{(D)}[27]$ & 2017 & V4, MiSeq, Dada2 & $61.03 \%$ & $81.72 \%$ & $3.82 \times 10^{4}$ & $3.3877 \times 10^{4}$ \\
PIH100 $^{(97)}[47]$ & 2020 & V12, MiSeq, FST.97 & $94.04 \%$ & $97.32 \%$ & $1.5740 \times 10^{4}$ & $1.3858 \times 10^{4}$ \\
PIH100 $^{(99)}[47]$ & 2020 & V12, MiSeq, FST.99 & $97.66 \%$ & $98.90 \%$ & $1.7351 \times 10^{4}$ & $1.5739 \times 10^{4}$ \\
PIH100 $^{(D)}[47]$ & 2020 & V12, MiSeq, Dada2 & $88.37 \%$ & $91.01 \%$ & $8.8630 \times 10^{3}$ & $9.0263 \times 10^{3}$ \\
Pseudomonas $^{(97)}$ & 2021 & V12, MiSeq, FST.97 & $97.49 \%$ & $98.92 \%$ & $3.0762 \times 10^{3}$ & $2.8235 \times 10^{3}$ \\
Pseudomonas $^{(99)}$ & 2021 & V12, MiSeq, FST.99 & $98.71 \%$ & $99.46 \%$ & $3.3810 \times 10^{3}$ & $3.0717 \times 10^{3}$ \\
Pseudomonas $^{(D)}$ & 2021 & V12, MiSeq, Dada2 & $89.26 \%$ & $93.77 \%$ & 1881.62 & 1889.10 \\
\hline
\end{tabular}

634 Table 1: Relative to study variables, within-genus taxa accumulation trends capture bulk 635 of the systematic variation in 16S surveys' genus-specific taxa accumulations. For each 636165 survey dataset mentioned in column 1, the year of publication is listed in column 2, the partial 637 16S segment targeted, machine technology and sequence clustering approach used are specified 638 in column 3. FST.x refers to sequence clustering at an a priori fixed sequence similarity threshold 639 of $\mathrm{x} \%$. McFadden's pseudo- $R^{2}$ for explaining genus-specific taxa accumulations with two 640 negative binomial regressions (NB) are listed in columns 4 and 5 . The fourth column is obtained 641 when the NB regression includes within-genus taxa accumulation trend $\left(\hat{f}_{R}(\cdot)\right.$, Methods) alone as 642 predictor. The fifth column additionally includes the genus identifier, total sample depth, and 643 experimental design matrix for each dataset as predictors (methods). Corresponding Akaike 644 Information Criteria (AIC) are listed in columns 6 and 7. 


\begin{tabular}{|l|l|l|l|l|l|l|}
\hline Dataset & Year & 16S segment, Sequencing\& Clustering & Pseudo $R_{\text {trend }}^{2}$ & Pseudo $R_{\text {trend }+ \text { design }}^{2}$ & $A_{\text {trend }}$ & $A I C_{\text {trend }+ \text { design }}$ \\
\hline Mouse [59, 60] & 2009 & V2, 454, FST.97 & $99.91 \%$ & $99.92 \%$ & $1.2971 \times 10^{3}$ & $1.2853 \times 10^{3}$ \\
Diarrhea [1] & 2014 & V12, 454, FST.99 & $99.94 \%$ & $99.94 \%$ & $1.2508 \times 10^{4}$ & $1.2406 \times 10^{4}$ \\
Time series [2] & 2014 & V4, GAIIx, FST.97 & $99.95 \%$ & $99.95 \%$ & $2.0972 \times 10^{3}$ & $1.9238 \times 10^{3}$ \\
Wastewater [48] $^{3}$ & 2018 & V34, MiSeq, FST.99 & $99.96 \%$ & $99.97 \%$ & $6.7384 \times 10^{2}$ & $6.3222 \times 10^{2}$ \\
MBQC-HLB $^{(97)}[27]$ & 2017 & V4, MiSeq, FST.97 & $99.98 \%$ & $99.99 \%$ & $2.5052 \times 10^{3}$ & $2.4587 \times 10^{3}$ \\
MBQC-HLB $^{(99)}[27]$ & 2017 & V4, MiSeq, FST.99 & $99.992 \%$ & $99.993 \%$ & $2.7456 \times 10^{3}$ & $2.6955 \times 10^{3}$ \\
MBQC-HLB $^{(D)}[27]$ & 2017 & V4, MiSeq, Dada2 & $99.86 \%$ & $99.03 \%$ & $1.6748 \times 10^{3}$ & $1.5003 \times 10^{3}$ \\
PIH100 $^{(97)}[47]$ & 2020 & V12, MiSeq, FST.97 & $98.96 \%$ & $99.07 \%$ & $1.2430 \times 10^{3}$ & $1.1989 \times 10^{3}$ \\
PIH100 $^{(99))}[47]$ & 2020 & V12, MiSeq, FST.99 & $99.94 \%$ & $99.95 \%$ & $1.4471 \times 10^{3}$ & $1.3988 \times 10^{3}$ \\
PIH100 $^{(D)}[47]$ & 2020 & V12, MiSeq, Dada2 & $99.73 \%$ & $99.73 \%$ & $9.8011 \times 10^{2}$ & $9.8415 \times 10^{2}$ \\
Pseudomonas $^{(97)}$ & 2021 & V12, MiSeq, FST.97 & $99.94 \%$ & $99.95 \%$ & $3.0641 \times 10^{2}$ & $2.8237 \times 10^{2}$ \\
Pseudomonas $^{(99)}$ & 2021 & V12, MiSeq, FST.99 & $99.97 \%$ & $99.98 \%$ & $3.1263 \times 10^{2}$ & $2.9741 \times 10^{2}$ \\
Pseudomonas $^{(D)}$ & 2021 & V12, MiSeq, Dada2 & $99.83 \%$ & $99.84 \%$ & $1.8723 \times 10^{2}$ & $1.9202 \times 10^{2}$ \\
\hline
\end{tabular}

Table 2: Relative to study variables, within-genus taxa accumulation trends capture bulk of the systematic variation in $16 \mathrm{~S}$ surveys' sample-wide taxa accumulations. For each $16 \mathrm{~S}$ survey dataset mentioned in column 1 , the year of publication is listed in column 2, the partial 16S segment targeted, machine technology and sequence clustering approach used are specified in column 3. FST.x refers to sequence clustering at an a priori fixed sequence similarity threshold of $x \%$. McFadden's pseudo- $R^{2}$ for explaining sample-wide taxa accumulations with two negative binomial regressions (NB) are listed in columns 4 and 5 . The fourth column is obtained when the

653 NB regression includes within-genus taxa accumulation trend $\left(\hat{f}_{R}(\cdot)\right.$, Methods) alone as predictor. 654 The fifth column additionally includes the total sample depth, and experimental design matrix for 655 each dataset as predictors (methods). Corresponding Akaike Information Criteria (AIC) are listed 656 in columns 6 and 7. 


\section{References}

658 1. Pop, M. et al. Diarrhea in young children from low-income countries leads to large-scale

659 alterations in intestinal microbiota composition. Genome Biol. 15, R76 (2014).

660 2. David, L. A. et al. Host lifestyle affects human microbiota on daily timescales. Genome Biol.

$661 \quad 15,1-15(2014)$.

662 3. Kostic, A. D. et al. The Dynamics of the Human Infant Gut Microbiome in Development and

663 in Progression toward Type 1 Diabetes. Cell Host Microbe 17, 260-273 (2015).

664 4. Riquelme, E. et al. Tumor Microbiome Diversity and Composition Influence Pancreatic

$665 \quad$ Cancer Outcomes. Cell 178, 795-806.e12 (2019).

666 5. Nejman, D. et al. The human tumor microbiome is composed of tumor type-specific

667 intracellular bacteria. Science 368, 973-980 (2020).

668 6. Poore, G. D. et al. Microbiome analyses of blood and tissues suggest cancer diagnostic 669 approach. Nature 579, 567-574 (2020).

670 7. Magurran, A. E. Ecological Diversity and Its Measurement. (Princeton University Press, 671 1988).

672 8. Magurran, A. E. \& McGill, B. J. Biological diversity: frontiers in measurement and

673 assessment. (Oxford University Press, 2011).

674 9. Hooper, D. U. et al. A global synthesis reveals biodiversity loss as a major driver of 675 ecosystem change. Nature 486, 105-108 (2012).

676 10. Purvis, A. \& Hector, A. Getting the measure of biodiversity. Nature 405, 212-219 (2000).

677 11. Fleishman, E., Noss, R. F. \& Noon, B. R. Utility and limitations of species richness metrics 678 for conservation planning. Ecol. Indic. 6, 543-553 (2006).

679 12. Adams, W. M., Small, R. D. S. \& Vickery, J. A. The impact of land use change on migrant 680 birds in the Sahel. Biodiversity 15, 101-108 (2014).

681 13. Hallmann, C. A., Foppen, R. P. B., van Turnhout, C. A. M., de Kroon, H. \& Jongejans, E. 682 Declines in insectivorous birds are associated with high neonicotinoid concentrations. 
Nature 511, 341-343 (2014).

14. Stanton, R. L., Morrissey, C. A. \& Clark, R. G. Analysis of trends and agricultural drivers of farmland bird declines in North America: A review. Agric. Ecosyst. Environ. 254, 244-254 (2018).

15. Inger, R. et al. Common European birds are declining rapidly while less abundant species' numbers are rising. Ecol. Lett. 18, 28-36 (2015).

16. Sambell, C. E., Holland, G. J., Haslem, A. \& Bennett, A. F. Diverse land-uses shape new bird communities in a changing rural region. Biodivers. Conserv. 28, 3479-3496 (2019).

17. Spellerberg, I. F. Monitoring Ecological Change. (Cambridge University Press, 2005). doi:10.1017/CBO9780511614699.

18. Adams, J. Species richness: patterns in the diversity of life. (Springer Science \& Business

19. Ozbudak, E. M., Thattai, M., Kurtser, I., Grossman, A. D. \& Van Oudenaarden, A. Regulation of noise in the expression of a single gene. Nat. Genet. 31, 69-73 (2002). Proc. Natl. Acad. Sci. 110, 3229-3236 (2013). the Microbiome. Conserv. Biol. 26, 195-197 (2012).

22. Jiménez, R. R. \& Sommer, S. The amphibian microbiome: natural range of variation,

23. West, A. G. et al. The microbiome in threatened species conservation. Biol. Conserv. 229, pathogenic dysbiosis, and role in conservation. Biodivers. Conserv. 26, 763-786 (2017).

24. Gotelli, N. J. \& Colwell, R. K. Quantifying biodiversity: procedures and pitfalls in the measurement and comparison of species richness. Ecol. Lett. 4, 379-391 (2001).

707 25. Gotelli, N. J. \& Colwell, R. K. Estimating species richness. 16.

708 26. Willis, A., Bunge, J. \& Whitman, T. Improved detection of changes in species richness in 
high diversity microbial communities. J. R. Stat. Soc. Ser. C Appl. Stat. 66, 963-977 (2017).

27. Sinha, R. et al. Assessment of variation in microbial community amplicon sequencing by the Microbiome Quality Control (MBQC) project consortium. Nat. Biotechnol. 35, 1077-1086

712 (2017).

28. Chao, A. \& Chiu, C.-H. Species Richness: Estimation and Comparison. in Wiley StatsRef: Statistics Reference Online 1-26 (American Cancer Society, 2016). doi:10.1002/9781118445112.stat03432.pub2.

29. Chao, A. Nonparametric Estimation of the Number of Classes in a Population. Scand. J. Stat. 11, 265-270 (1984).

30. Bunge, J. \& Fitzpatrick, M. Estimating the Number of Species: A Review. J. Am. Stat. Assoc. 88, 364-373 (1993).

31. Bent, S. J. \& Forney, L. J. The tragedy of the uncommon: understanding limitations in the analysis of microbial diversity. ISME J. 2, 689-695 (2008).

32. Kunin, V., Engelbrektson, A., Ochman, H. \& Hugenholtz, P. Wrinkles in the rare biosphere: pyrosequencing errors can lead to artificial inflation of diversity estimates. Environ.

33. Huse, S. M., Welch, D. M., Morrison, H. G. \& Sogin, M. L. Ironing out the wrinkles in the rare biosphere through improved OTU clustering. Environ. Microbiol. 12, 1889-1898 (2010).

34. Schloss, P. D. Reintroducing mothur: 10 Years Later. Appl. Environ. Microbiol. 86, (2020).

35. Chiu, C.-H. \& Chao, A. Estimating and comparing microbial diversity in the presence of sequencing errors. PeerJ 4, e1634 (2016).

36. Willis, A. Species richness estimation with high diversity but spurious singletons. (2016). identity may not be sufficient to guarantee species identity. Int. J. Syst. Evol. Microbiol. 42, $166-170$ (1992).

38. Janda, J. M. \& Abbott, S. L. 16S rRNA Gene Sequencing for Bacterial Identification in the 
Diagnostic Laboratory: Pluses, Perils, and Pitfalls. J. Clin. Microbiol. 45, 2761-2764 (2007).

39. Knight, R. et al. Best practices for analysing microbiomes. Nat. Rev. Microbiol. 16, 410-422 (2018).

40. Hillmann, B. et al. Evaluating the Information Content of Shallow Shotgun Metagenomics.

$$
\text { mSystems 3, (2018). }
$$

41. Johnson, J. S. et al. Evaluation of $16 \mathrm{~S}$ rRNA gene sequencing for species and strain-level

741 microbiome analysis. Nat. Commun. 10, 5029 (2019).

742 42. Yarza, P. et al. Uniting the classification of cultured and uncultured bacteria and archaea

743 using 16S rRNA gene sequences. Nat. Rev. Microbiol. 12, 635-645 (2014).

744 43. Větrovskỳ, T. \& Baldrian, P. The variability of the 16 S rRNA gene in bacterial genomes and

745 its consequences for bacterial community analyses. PloS One 8, e57923 (2013).

746 44. Poretsky, R., Rodriguez-R, L. M., Luo, C., Tsementzi, D. \& Konstantinidis, K. T. Strengths and Limitations of 165 rRNA Gene Amplicon Sequencing in Revealing Temporal Microbial Community Dynamics. PLOS ONE 9, e93827 (2014).

45. Harris, B. Determining bounds on integrals with applications to cataloging problems. Ann. Math. Stat. 521-548 (1959).

46. Gelman, A. Struggles with Survey Weighting and Regression Modeling. Stat. Sci. 22, (2007).

47. Fisher, R. A., Corbet, A. S. \& Williams, C. B. The Relation Between the Number of Species and the Number of Individuals in a Random Sample of an Animal Population. J. Anim. Ecol. $12,42-58$ (1943).

48. Chao, A. \& Lee, S.-M. Estimating the Number of Classes via Sample Coverage. J. Am. Stat. Assoc. 87, 210-217 (1992). (2015).

50. Paulson, J. N. et al. Paenibacillus infection with frequent viral coinfection contributes to 
postinfectious hydrocephalus in Ugandan infants. Sci. Transl. Med. 12, (2020).

51. Kulkarni, P. et al. Conventional wastewater treatment and reuse site practices modify bacterial community structure but do not eliminate some opportunistic pathogens in reclaimed water. Sci. Total Environ. 639, 1126-1137 (2018).

52. Degnan, P. H. \& Ochman, H. Illumina-based analysis of microbial community diversity. ISME J. 6, 183-194 (2012).

53. Pinto, A. J. \& Raskin, L. PCR biases distort bacterial and archaeal community structure in pyrosequencing datasets. (2012).

54. Schloss, P. D., Gevers, D. \& Westcott, S. L. Reducing the effects of PCR amplification and sequencing artifacts on 16S rRNA-based studies. PloS One 6, e27310 (2011).

55. Haas, B. J. et al. Chimeric 16S rRNA sequence formation and detection in Sanger and 454pyrosequenced PCR amplicons. Genome Res. 21, 494-504 (2011). Taxonomic Unit-Based Approaches for 16S rRNA Gene Sequence Analysis. Appl. Environ. Microbiol. 77, 3219-3226 (2011).

57. Sze, M. A. \& Schloss, P. D. The Impact of DNA Polymerase and Number of Rounds of Amplification in PCR on 16S rRNA Gene Sequence Data. mSphere 4, (2019). Uncountable: Statistical Approaches to Estimating Microbial Diversity. Appl. Environ. Microbiol. 67, 4399-4406 (2001).

59. Olson, N. D. et al. A framework for assessing 16S rRNA marker-gene survey data analysis methods using mixtures. Microbiome 8, 1-18 (2020).

60. Prodan, A. et al. Comparing bioinformatic pipelines for microbial $16 \mathrm{~S}$ rRNA amplicon sequencing. PLOS ONE 15, e0227434 (2020).

61. Haegeman, B. et al. Robust estimation of microbial diversity in theory and in practice. ISME J. 7, 1092-1101 (2013). 
62. Flather, C. Fitting species-accumulation functions and assessing regional land use impacts on avian diversity. J. Biogeogr. 23, 155-168 (1996).

63. Goeman, J. J. \& Finos, L. The inheritance procedure: multiple testing of tree-structured hypotheses. Stat. Appl. Genet. Mol. Biol. 11, (2012).

64. Meijer, R. J. \& Goeman, J. J. A multiple testing method for hypotheses structured in a directed acyclic graph. Biom. J. 57, 123-143 (2015).

65. Gu, C. Smoothing spline ANOVA models. (Springer, 2002).

66. Gu, C. Smoothing spline ANOVA models. vol. 297 (Springer Science \& Business Media, 2013).

67. DiCiccio, T. J. \& Efron, B. Bootstrap confidence intervals. Stat. Sci. 11, 189-228 (1996).

68. Oksanen, J. et al. The vegan package. Community Ecol. Package 10, 719 (2007).

69. Hsieh, T. C., Ma, K. H. \& Chao, A. iNEXT: an R package for rarefaction and extrapolation of species diversity (H ill numbers). Methods Ecol. Evol. 7, 1451-1456 (2016).

70. Weston, S. \& Calaway, R. Getting Started with doParallel and foreach. Vignette CRAN URL 957, (2019).

71. Chang, S.-S., Hsu, H.-L., Cheng, J.-C. \& Tseng, C.-P. An efficient strategy for broad-range detection of low abundance bacteria without DNA decontamination of PCR reagents. PloS One 6, e20303 (2011).

72. Paulson, J. N. et al. metagenomeSeq: Statistical analysis for sparse high-throughput sequencing. (Bioconductor version: Release (3.13), 2021). doi:10.18129/B9.bioc.metagenomeSeq.

73. Paulson, J. N., Bravo, H. C., Pop, M. \& biocViews ExperimentData, S. Package 'msd16s'. (2015).

74. Bolger, A. M., Lohse, M. \& Usadel, B. Trimmomatic: a flexible trimmer for Illumina sequence data. Bioinformatics 30, 2114-2120 (2014).

75. Callahan, B. J. et al. DADA2: high-resolution sample inference from Illumina amplicon data. 
Nat. Methods (2016).

814 76. Martin, M. Cutadapt removes adapter sequences from high-throughput sequencing reads.

$815 \quad$ EMBnet J. 17, 10-12 (2011).

816 77. Caporaso, J. G. et al. QIIME allows analysis of high-throughput community sequencing

817 data. Nat. Methods 7, 335-336 (2010).

818 78. Zhang, J., Kobert, K., Flouri, T. \& Stamatakis, A. PEAR: a fast and accurate Illumina Paired-

819 End reAd mergeR. Bioinformatics 30, 614-620 (2014).

820 79. Edgar, R. C. Search and clustering orders of magnitude faster than BLAST. Bioinforma. Oxf.

$821 \quad$ Engl. 26, 2460-2461 (2010).

822 80. Quast, C. et al. The SILVA ribosomal RNA gene database project: improved data

823 processing and web-based tools. Nucleic Acids Res. 41, D590-D596 (2013).

824 81. Caporaso, J. G. et al. PyNAST: a flexible tool for aligning sequences to a template

825 alignment. Bioinformatics 26, 266-267 (2010). 